adalimumab tapering at 6 months' remission and 2 cases initiated adalimumab tapering at 9-12 months' remission. No patients had recurrent uveitis at 12 months' remission or longer in both groups. Adalimumab plus MTX were well tolerated in all patients.

Conclusions: For maintaining remission of SpA-related uveitis, adalimumab tapering can be effective when combined with MTX. Initiation of the tapering after 12 months' remission largely lower the rate of recurrence.

References:

[1] Jaffe GJ, Dick AD, Brézin AP, et al. Adalimumab in Patients with Active Noninfectious Uveitis. N Engl J Med. 2016 Sep 8; 375(10):932-43.

Disclosure of Interest: None declared

DOI: 10.1136/annrheumdis-2017-eular.2507

\section{THU0352 TOFACITINIB TREATMENT IS ASSOCIATED WITH ATTAINMENT OF THE MINIMALLY IMPORTANT REDUCTION IN AXIAL MRI INFLAMMATION IN PATIENTS WITH ANKYLOSING} SPONDYLITIS

W.P. Maksymowych ${ }^{1}$, D. van der Heijde ${ }^{2}$, X. Baraliakos ${ }^{3}$, A. Deodhar ${ }^{4}$ M. Brown ${ }^{5}$, S.P. Sherlock ${ }^{6}$, D. Li ${ }^{7}$, D. Fleishaker ${ }^{8}$, T. Hendrikx ${ }^{7}$, K.S. Kanik ${ }^{8}$.

1 University of Alberta, Edmonton, AB, Canada; ${ }^{2}$ Leiden University Medical Center, Leiden, Netherlands; ${ }^{3}$ Rheumazentrum Ruhrgebiet, Herne, Germany;

${ }^{4}$ Oregon Health \& Science University, Portland, OR, United States; ${ }^{5}$ Queensland University of Technology, Brisbane, Australia; ${ }^{6}$ Pfizer Inc, Cambridge, MA; ${ }^{7}$ Pfizer Inc, Collegeville, PA; ${ }^{8}$ Pfizer Inc, Groton, CT, United States

Background: Tofacitinib is an oral Janus kinase inhibitor. The minimally important changes (MICs) for the SPondyloArthritis Research Consortium of Canada (SPARCC) MRI sacroiliac joint (SIJ) and spine scores based on agreement with global change scores by readers are $\geq 2.5$ and $\geq 5$, respectively.

Objectives: To assess whether MIC in SIJ and spine can discriminate between tofacitinib and placebo (PBO) in patients (pts) with ankylosing spondylitis (AS) and if this is concordant with clinical responses.

Methods: In this 16-week (wk), Phase 2, double-blind, dose-ranging study (NCT01786668), ${ }^{2} 207$ adult pts meeting modified New York AS criteria were randomised 1:1:1:1 to PBO or tofacitinib 2, 5 or $10 \mathrm{mg}$ twice daily (BID) for 12 wks. Clinical endpoints included in this post-hoc analysis were: Assessment of SpondyloArthritis International Society 20\% improvement (ASAS20) and ASAS $40 \%$ improvement (ASAS40) response rates, AS disease activity score major improvement (ASDAS MI; change $\geq 2.0$ from baseline), ASDAS inactive disease (ASDAS ID; <1.3), Bath AS disease activity index (BASDAI), Bath AS functional index (BASFI) and back pain. Pts (\%) achieving MIC in SIJ, spine and both SIJ and spine, in tofacitinib and PBO groups, were summarised based on observed data, and pooled tofacitinib ( 5 and $10 \mathrm{mg} \mathrm{BID}$ ) vs PBO data were compared using Fisher's exact test. Concordance between achieving MIC and Wk 12 clinical responses was assessed. Wk 12 clinical responses were compared between pts achieving/not achieving MIC.

Results: MRI data for 164 pts were evaluated. Baseline demographics were generally balanced between treatment groups and typical of AS populations. ${ }^{2}$ Tofacitinib 2, 5 and $10 \mathrm{mg} \mathrm{BID}$ improved mean (range) SPARCC scores vs PBO (SIJ: $-2.2[-22.0,10.5],-3.5[-34.5,11.0],-3.6[-29.0,0.5]$ vs $-0.7[-9.5,6.5]$; spine: $-3.2[-34.5,20.5],-5.5[-36.5,8.0],-6.7[-32.5,7.5]$ vs $-0.8[-8.0,14.0])$. Approximately 3 times more pts achieved MIC in SIJ or spine in the pooled tofacitinib group vs PBO (SIJ: $34.1 \%$ vs $11.8 \%, p<0.05$; spine: $38.6 \%$ vs $11.8 \%$, $p<0.01)$. Achieving MIC in SIJ and spine correlated with clinical response. In pts on tofacitinib, ASAS20, ASAS40 and ASDAS MI responses were more likely in pts achieving MIC in SIJ or spine (Table) vs not achieving MIC. Compared with not achieving MIC, pts on tofacitinib achieving MIC in SIJ had larger improvements in BASDAI, BASFI and back pain

Conclusions: Pts who received tofacitinib who had AS experienced clinically meaningful reductions in axial MRI inflammation. Pts achieving MIC for MRI inflammation had increased clinical response rates.

\section{References:}

[1] Maksymowych WP et al. J Rheumatol 2012; 39: 1666-1674.

[2] van der Heijde D et al. Ann Rheum Dis 2016; In press.

Acknowledgements: Previously presented at ACR 2016 and reproduced with permission. This study was sponsored by Pfizer Inc. Editorial support was provided by A Pedder of CMC and funded by Pfizer Inc.

Disclosure of Interest: W. Maksymowych Grant/research support from: AbbVie, Pfizer Inc, Sanofi, UCB, Consultant for: AbbVie, Amgen, Eli Lilly, Janssen, Merck, Novartis, Pfizer Inc, Sanofi, UCB, D. van der Heijde Consultant for: AbbVie, Amgen, Astellas, AstraZeneca, Bristol-Meyers Squibb, Boehringer Ingelheim, Celgene, Daiichi, Eli Lilly, Galapagos, Janssen, Merck, Novartis, Pfizer Inc, Roche, Sanofi-Aventis, UCB, Employee of: Imaging Rheumatology BV, X. Baraliakos Grant/research support from: AbbVie, Bristol-Myers Squibb, Celgene, Janssen, Novartis, Pfizer Inc, Roche, MSD, UCB, A. Deodhar Grant/research support from: AbbVie, Amgen, Eli Lilly, Janssen, Novartis, Pfizer Inc, UCB, Speakers bureau: AbbVie, Amgen, Boehringer Ingelheim, Janssen, Novartis, Pfizer Inc, UCB, M. Brown Grant/research support from: AbbVie, Janssen, Leo Pharma, UCB, Consultant for: AbbVie, Janssen, Novartis, Pfizer Inc, UCB, Speakers bureau: AbbVie, Janssen, Novartis, Pfizer Inc, UCB, S. Sherlock Shareholder of: Pfizer Inc, Employee of: Pfizer Inc, D. Li Shareholder of: Pfizer Inc, Employee of: Pfizer Inc, D. Fleishaker Shareholder of: Pfizer Inc, Employee of: Pfizer Inc, T. Hendrikx

\begin{tabular}{|c|c|c|c|c|}
\hline & $\begin{array}{l}\text { ASAS20 } \\
\text { n/N (\%) }\end{array}$ & $\begin{array}{l}\text { ASAS40 } \\
\text { n N (\%) }\end{array}$ & $\begin{array}{c}\text { ASDAS MII } \\
\mathbf{n} / \mathbf{N}(\%)\end{array}$ & $\begin{array}{l}\text { ASDAS ID } \\
\text { n/N (\%) }\end{array}$ \\
\hline \multicolumn{5}{|l|}{ Placebo } \\
\hline $\mathrm{SU} \geq \mathrm{MIC}(2.5)$ & $3 / 4(75.0)$ & $1 / 4(25.0)$ & $0 / 4$ & $0 / 4$ \\
\hline $\mathrm{SIJ}<\mathrm{MIC}(2.5)$ & $14 / 29(48.3)$ & $6 / 29(20.7)$ & $3 / 28(10.7)$ & $3 / 28(10.7)$ \\
\hline Spine $\geq \operatorname{MIC~(5)~}$ & $3 / 4(75.0)$ & $2 / 4(50.0)$ & $2 / 4(50.0)$ & $1 / 4(25.0)$ \\
\hline Spine $<\mathrm{MIC}(5)$ & $14 / 29(48.3)$ & $5 / 29(17.2)$ & $1 / 28(3.6)$ & $2 / 28(7.1)$ \\
\hline \multicolumn{5}{|c|}{ Tofacitinib 2 mg BID } \\
\hline $\mathrm{SUI} \geq \mathrm{MIC}(2.5)$ & $9 / 12(75.0)$ & $7 / 12(58.3)$ & $5 / 12(41.7)$ & $2 / 12(16.7)$ \\
\hline SIJ $<$ MIC $(2.5)$ & $15 / 29(51.7)$ & $12 / 29(41.4)$ & $3 / 30(10.0)$ & $4 / 30(13.3)$ \\
\hline Spine $\geq \operatorname{MIC}(5)$ & $11 / 12(91.7)$ & $8 / 12(66.7)$ & $4 / 12(33.3)$ & $3 / 12(25.0)$ \\
\hline Spine $<\mathrm{MIC}(5)$ & $13 / 28(46.4)$ & $11 / 28(39.3)$ & $4 / 29(13.8)$ & $3 / 29(10.3)$ \\
\hline \multicolumn{5}{|c|}{ Tofacitinib 5 mg BID } \\
\hline $\mathrm{SIJ} \geq \mathrm{MIC}(2.5)$ & $15 / 17(88.2)$ & $10 / 17(58.8)$ & $6 / 17(35.3)$ & $2 / 17(11.8)$ \\
\hline SIJ $<\mathrm{MIC}(2.5)$ & $21 / 25(84.0)$ & $12 / 25(48.0)$ & $5 / 26(19.2)$ & $5 / 26(19.2)$ \\
\hline Spine $\geq$ MIC (5) & $12 / 14(85.7)$ & $8 / 14(57.1)$ & $5 / 15(33.3)$ & $3 / 15(20.0)$ \\
\hline Spine $<\mathrm{MIC}(5)$ & $24 / 28(85.7)$ & $14 / 28(50.0)$ & $6 / 28(21.4)$ & $4 / 28(14.3)$ \\
\hline \multicolumn{5}{|c|}{ Tofacitinib $10 \mathrm{mg} \mathrm{BID}$} \\
\hline $\mathrm{SIJ} \geq \mathrm{MIC}(2.5)$ & $9 / 13(69.2)$ & $8 / 13(61.5)$ & $4 / 13(30.8)$ & $4 / 13(30.8)$ \\
\hline $\mathrm{SIJ} \triangleleft \mathrm{MIC}(2.5)$ & $18 / 31(58.1)$ & $12 / 31(38.7)$ & $9 / 31(29.0)$ & 4/31 (12.9) \\
\hline Spine $\geq \operatorname{MIC~(5)~}$ & $13 / 18(72.2)$ & $10 / 18(55.6)$ & $7 / 18(38.9)$ & 2/18(11.1) \\
\hline Spine $<$ MIC (5) & $14 / 26(53.9)$ & $10 / 26(38.5)$ & $6 / 26(23.1)$ & $6 / 26(23.1)$ \\
\hline \multicolumn{5}{|c|}{ Pooled tofacitinib 5 and $10 \mathrm{mg} \mathrm{BID}$} \\
\hline $\mathrm{SIJ} \geq \mathrm{MIC}(2.5)$ & $24 / 30(80.0)$ & $18 / 30(60.0)$ & $10 / 30(33.3)$ & $6 / 30(20.0)$ \\
\hline $\mathrm{SUJ}<\mathrm{MIC}(2.5)$ & $39 / 56(69.6)$ & $24 / 56(42.9)$ & $14 / 57(24.6)$ & 9/57 (15.8) \\
\hline Spine $\geq \operatorname{MCC}(5)$ & $25 / 32(78.1)$ & $18 / 32(56.3)$ & $12 / 33(36.4)$ & $5 / 33(15.2)$ \\
\hline Spine $<M I C(5)$ & $38 / 54(70.4)$ & $24 / 54(44.4)$ & $12 / 54(22.2)$ & $10 / 54(18.5)$ \\
\hline
\end{tabular}

Shareholder of: Pfizer Inc, Employee of: Pfizer Inc, K. Kanik Shareholder of: Pfizer Inc, Employee of: Pfizer Inc

DOI: 10.1136/annrheumdis-2017-eular.2447

\section{THU0353 CHANGE IN MRI STRUCTURAL LESIONS IN THE SACROILIAC JOINT AFTER TWO YEARS OF ETANERCEPT THERAPY IN COMPARISON TO A CONTEMPORARY CONTROL COHORT IN NON-RADIOGRAPHIC AXIAL SPONDYLOARTHRITIS}

W.P. Maksymowych ${ }^{1}$, M. Dougados ${ }^{2}$, R.G. Lambert ${ }^{1}$, R. Landewe ${ }^{3}$, A. Molto $^{2}$, P. Claudepierre ${ }^{4}$, M. de Hooge ${ }^{5}$, R. Bonin ${ }^{6}$, J.F. Bukowski ${ }^{6}$, H. Jones ${ }^{6}$,

I. Logeart ${ }^{7}$, R. Pedersen ${ }^{6}$, A. Szumski ${ }^{8}$, B. Vlahos $^{6}$, D. van der Heijde ${ }^{5}$

${ }^{1}$ University of Alberta, Edmonton, Canada; ${ }^{2}$ Paris Descartes University, Hôpital Cochin, Paris, France; ${ }^{3}$ Amsterdam Rheumatology \& Immunology Center, Amsterdam, Netherlands; ${ }^{4}$ Universite Paris Est Creteil, Creteil, France; ${ }^{5}$ Leiden University Medical Center, Leiden, Netherlands; ${ }^{6}$ Pfizer, Collegeville, United States; ${ }^{7}$ Pfizer, Paris, France, ${ }^{8}$ InVentiv Health, Princeton, United States

Background: Demonstrating a structural effect of TNF inhibitors in axial SpA (axSpA) is challenging.

Objectives: To compare 2 yrs of structural lesion changes on T1W MRI in the sacroiliac joints (SIJ) of pts receiving etanercept (ETN) in a clinical trial to similar pts not receiving biologics in a cohort study.

Methods: Pts had recent onset non-radiographic (nr)-axSpA fulfilling ASAS criteria. Study group: pts receiving ETN $50 \mathrm{mg}$ once weekly for 2 yrs in EMBARK (NCT01258738). Control group: pts in a longitudinal cohort study not receiving biologics for 2 yrs (DESIR, NCT01648907). Outcome measure: change in structural lesions of erosion, backfill, fat metaplasia, and ankylosis. MRI images were read by 3 experienced readers unaware of image chronology and pt group, using the SpondyloArthritis Research Consortium of Canada SIJ Structural Score (SSS). ${ }^{1}$ For each group, differences were calculated between percentages of patients experiencing increases and decreases in structural lesion scores over 2

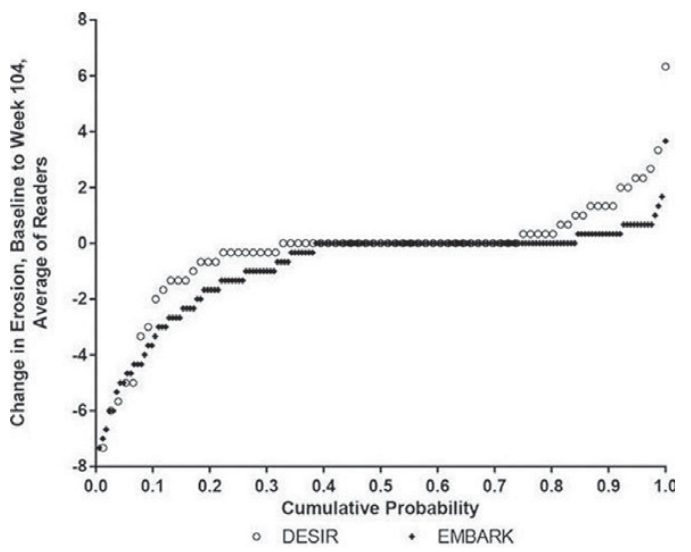

Figure 1. Cumulative Probability of Change in MRI SIJ Erosion, BL to Week 104. 